\title{
Evaluation of Plasma Assisted Noninvasive Surgery (PANIS) As a New Approach for the Treatment of Conjunctival Concretions: A Clinical Case Series
}

\author{
Farhad Nejat ${ }^{* 1}$, Khosrow Jadidi ${ }^{1}$, Shima Eghtedari ${ }^{1}$, Nazanin-Sadat Nabavi ${ }^{1}$, Pooneh Malekifar ${ }^{1,2}$ \\ ${ }^{1}$ Vision Health Research Center, Tehran, Iran \\ ${ }^{2} \mathrm{PhD}$ of Epidemiology, Department of Epidemiology, School of Public Health, Tehran University Medical Sciences, \\ Tehran, Iran
}

*Corresponding Author: Farhad Nejat; Vision Health Research Center, Tehran, Iran; fanejat@ yahoo.com

Received 16 November 2021;

Accepted 30 November 2021;

Published 05 December 2021

\begin{abstract}
Background and Objective: Plasma is one of the newest therapies recently used. This study aimed to evaluate a quick treatment modality of conjunctival concretions using plasma as a noninvasive procedure. Methods: Six eyes of six patients with conjunctival concretions were evaluated. The procedure was performed using plasma spots generated by the white handpiece of the Plexr device. The eyelid was everted and concretions sublimated by plasma spots from the upper and lower eyelids. Refractive Errors, visual acuity (BCVA and UCVA), Ocular Surface Disease Index (OSDI), and Intraocular pressure (IOP) were measured before and after six months of the procedure. $\underline{\text { Results: }}$ The results of 6 months follow-up show that this effective method had no recurrence or side effects. Treatment was effective in all of the patients. No complication and recurrence was observed. The OSDI score was improved to the normal range. This method had no effects on Refractive Errors, Visual Acuity, and IOP. Conclusion: Based on the results, it seems that the PANIS method can be used as a practical, quick, and straightforward approach for the treatment of conjunctival concretions.
\end{abstract}

Keywords: conjunctival concretions, plasma, PANIS, case series

\section{Introduction}

Conjunctival concretions are single or multiple benign entities with various sizes and yellowish-white in color. They represent the residues of leucocytes' degenerative products, which are seen through the superficial or in-depth of the conjunctival epithelium ${ }^{[1]}$. It is often idiopathic and occurs in the elderly but is sometimes secondary to people with chronic inflammatory conjunctival diseases such as trachoma, vernal keratoconjunctivitis, atopic keratoconjunctivitis, meibomian glandular disease, and dry eye ${ }^{[2]}$. Therefore, there is a chance of diagnosing it in young people. Even though few studies have been done in this field, its prevalence has been reported up to $40 \%{ }^{[1,3]}$. This disease is often asymptomatic, but prominent concretions cause abrasion, pain, redness, photophobia, blurred vision, and corneal ulceration ${ }^{[4]}$. The eye specialist usually makes a diagnosis with everting the upper and lower eyelids. In cases where conjunctival concretions cause annoying complaints, these concretions need to be removed by local anesthesia (e.g., topical tetracaine $0.5 \%$ ) with a $25 \mathrm{G}$ sterile needle behind the slit lamp ${ }^{[4,5]}$. Rarely, in cases where there are a lot and large concretions, surgery is performed in the operating room and under anesthesia. In general, there is not much variety in surgical methods for this disease, and most patients are treated as an office base method with a needle, which is almost aggressive. One of the new therapies that have been considered in recent years is the use of the beneficial properties of Plasma ${ }^{[6]}$. First time in 2013, Plasma was used to stimulate tissue regeneration as well as against microbial pathogens ${ }^{[7]}$. In animal studies, Nejat et al. confirmed the use of atmospheric low-temperature plasma (ALTP) on the surface of rabbit eyes ${ }^{[8,9]}$. Also, the effectiveness of the PANIS method in treating Conjunctivochalasis, Dry eye disease, Pinguecula and Conjunctival Cyst has been shown in human studies ${ }^{[10-13]}$.

In this study, for the first time, we intend to use this method to treat patients with conjunctival concretions and examine its possible side effects as a new method.

\section{Case Reports}

\section{Patients}

All candidates with symptomatic concretions were examined with a complete ophthalmic examination to confirm of disease. In this 
study, six patients ( 2 females and four males) participated with a mean age of $53.83 \pm 6.49$. Three participants had their right eye, and three had their left eye involved. Concretions were upper eyelid in three eyes and lower eyelid in three eyes. Three patients had a single concretion, and three patients had multiple concretions. We evaluated ophthalmic parameters before and after six months of the procedure. We measured Refractive Errors, visual acuity (BCVA and UCVA), Ocular Surface Disease Index (OSDI), and Intraocular pressure (IOP). IOP was measured by using a rebound tonometer (Icare Finland Oy, Vantaa, Finland). The OSDI questionnaire consists of 12 questions. The final score was $0-100$, which the higher score considered as a more dry eye. We had two self-reported questions for measuring satisfaction in patients. The first question was if you will face this disease in your other eye, would you rather treat it with the PANIS method or not? And the second one was would you inform your relatives about the PANIS method for treating their similar eye disease?

Plasma assisted noninvasive surgery (PANIS Method)
In this study, we evaluated the PANIS method for concretions treatment. All of the procedure is done by one surgeon (Dr. Nejat F). Before treatment, topical tetracaine $0.5 \%$ eye drop (Sina Daro, Tehran, Iran) was used to numb the eye. We used This drop every 5 minutes and 5 minutes after the third drop; patients at the slitlamp treated using a white handpiece of the Plexr device ( Vpp = $500 \mathrm{~V}$, Power $=0.7 \mathrm{~W}$, and Frequency $=75 \mathrm{kHz}$ ). More information about the technical features of the Plexr device is in table 1 . The eyelid everted and concretions sublimated with one or two plasma spots from the upper and lower eyelids. This process continues until the tissue of the concretions is completely sublimated. (See supplemental video). To prevent recurrence in cases with a considerable cavity under the concretions, we apply to the bottom of the hole. After treatment, ciprofloxacin $0.3 \%$ (Sina Darou, Tehran, Iran) every 6 hours for one week and Artificial tear (like Tearlose $10 \mathrm{ml}$ (Sina Darou, Tehran, Iran) every 8 hours for three months, were prescribed. In cases where concretions were seen as secondary, we treated the cause of the disease to prevent a recurrence.

Table 1: Technical features of the Plexr device.

\begin{tabular}{|l|l|}
\hline Parameters & Values \\
\hline Working gas Air & Air \\
\hline Power supply & Docking station $=24 \mathrm{~V}$ \\
\hline & Hand pieces: embedded inductive charger $=5 \mathrm{~V}$ \\
\hline Hand pieces: & \\
\hline Max output & $\leq 2 \mathrm{~W}$ \\
\hline Max working voltage & $\leq 1.3 \mathrm{kVPP}$ \\
\hline Output frequency & $(70-80) \mathrm{kHz}$ \\
\hline Hand piece types: & \\
\hline White* & V peak to peak $=500 \mathrm{~V}$, Power $=0.7 \mathrm{~W}$, Frequency $=75 \mathrm{kHz}$ \\
\hline Green & V peak to peak $=600$ V, Power $=1 \mathrm{~W}$, Frequency $=75 \mathrm{kHz}$ \\
\hline Red & V peak to peak $=700 \mathrm{~V}$, Power $=2 \mathrm{~W}$, Frequency $=75 \mathrm{kHz}$ \\
\hline Maximum absorbed power & $120 \mathrm{~W}$ \\
\hline (Docking station) & \\
\hline Applicator electrode & Stainless steel sterile disposable needle \\
\hline Risk classification of the device & Ilb**(Medium-high risk) \\
\hline $\begin{array}{l}* \text { In current study, the white hand piece was used. } \\
* * \text { This classification relates to the Non-invasive medical devices within the field of dermatology. }\end{array}$ \\
\hline
\end{tabular}

\section{Results of treatment}

Based on the results of this study, patients treated with the PANIS method were satisfied. According to the OSDI index, after treatment, the OSDI score improved to the normal range, and in two patients, it improved to mild. (Table 2) According to our results, treatment had no effects on the patient's vision. BCVA and UCVA for each patient were almost the same before and after the procedure, so visual acuity had not changed. As expected, this method had no effects on refractive errors measures. (Table 2) Before the procedure, all of the cases had normal Intraocular pressure (IOP). After that, there were no high-risk increases in IOP, and in some cases, the IOP is the same or reduced. There were no intraoperative and postoperative complications. Results showed that all of the patients had no relapse after six months, and treatment had optimal results. (Figure 1) Reached from two questions for measuring satisfaction in patients, all of the patients highly recommended this method to others, and if they had this disease in another eye, they would use this approach for treatment.

Table 2: The ophthalmic parameters before and 6 month after procedure

\begin{tabular}{|c|c|c|c|c|c|c|c|c|c|c|c|c|c|}
\hline \multirow[t]{3}{*}{ patient } & \multirow[t]{3}{*}{ Type } & \multirow[t]{3}{*}{ Age } & \multirow[t]{3}{*}{ Eye } & \multirow[t]{3}{*}{ Time } & & & & & & & & & \\
\hline & & & & & \multirow{2}{*}{\multicolumn{3}{|c|}{ Refractive Errors }} & \multicolumn{2}{|c|}{ Visual Acuity } & \multirow[t]{2}{*}{ IOP } & \multirow[t]{2}{*}{ OSDI } & \multirow[t]{2}{*}{ Q1 } & \multirow[t]{2}{*}{ Q2 } \\
\hline & & & & & & & & UCVA & BCVA & & & & \\
\hline Patient & multiple & 46 years & OS & Pre-procedure & -1 & -0.75 & 180 & 0.6 & 1 & 16 & 80 & & \\
\hline 1 & concretions & old & Inferior & Post- procedure & -0.75 & -0.75 & 175 & 0.6 & 1 & 14 & 16 & YES & YES \\
\hline Patient & single & 62 years & OD & Pre-procedure & Plano & -0.5 & 75 & 1 & 1 & 15 & 84 & & \\
\hline 2 & concretion & old & Superior & Post- procedure & -0.5 & -0.5 & 85 & 1 & 1 & 15 & 21 & YES & YES \\
\hline Patient & single & 61 years & OD & Pre-procedure & -0.5 & Plano & Plano & 0.9 & 1 & 18 & 45 & & \\
\hline 3 & concretion & old & Superior & Post- procedure & -0.5 & -0.5 & 157 & 0.9 & 1 & 17 & 12 & YES & YES \\
\hline
\end{tabular}




\begin{tabular}{|c|c|c|c|c|c|c|c|c|c|c|c|c|c|}
\hline $\begin{array}{c}\text { Patient } \\
4\end{array}$ & $\begin{array}{c}\text { multiple } \\
\text { concretions }\end{array}$ & $\begin{array}{c}51 \text { years } \\
\text { old }\end{array}$ & $\begin{array}{c}\text { OS } \\
\text { Superior }\end{array}$ & $\begin{array}{l}\text { Pre-procedure } \\
\text { Post- procedure }\end{array}$ & $\begin{array}{l}-0.5 \\
-0.25\end{array}$ & $\begin{array}{l}-1.25 \\
-1.25\end{array}$ & $\begin{array}{l}178 \\
172\end{array}$ & $\begin{array}{l}0.7 \\
0.7\end{array}$ & $\begin{array}{l}1 \\
1\end{array}$ & $\begin{array}{l}13 \\
15\end{array}$ & $\begin{array}{l}63 \\
10\end{array}$ & YES & YES \\
\hline Patient & single & 49 years & OD & Pre-procedure & -1 & -0.75 & 80 & 0.6 & 1 & 12 & 68 & & \\
\hline 5 & concretion & old & Inferior & Post- procedure & -0.25 & 0.25 & 79 & 0.6 & 1 & 14 & 27 & YES & YES \\
\hline Patient & multiple & 54 years & OS & Pre-procedure & -2.5 & -3.5 & 11 & 0.1 & 1 & 15 & 80 & & \\
\hline 6 & concretions & old & Inferior & Post- procedure & -2 & -3.75 & 15 & 0.1 & 1 & 15 & 9 & YES & YES \\
\hline
\end{tabular}
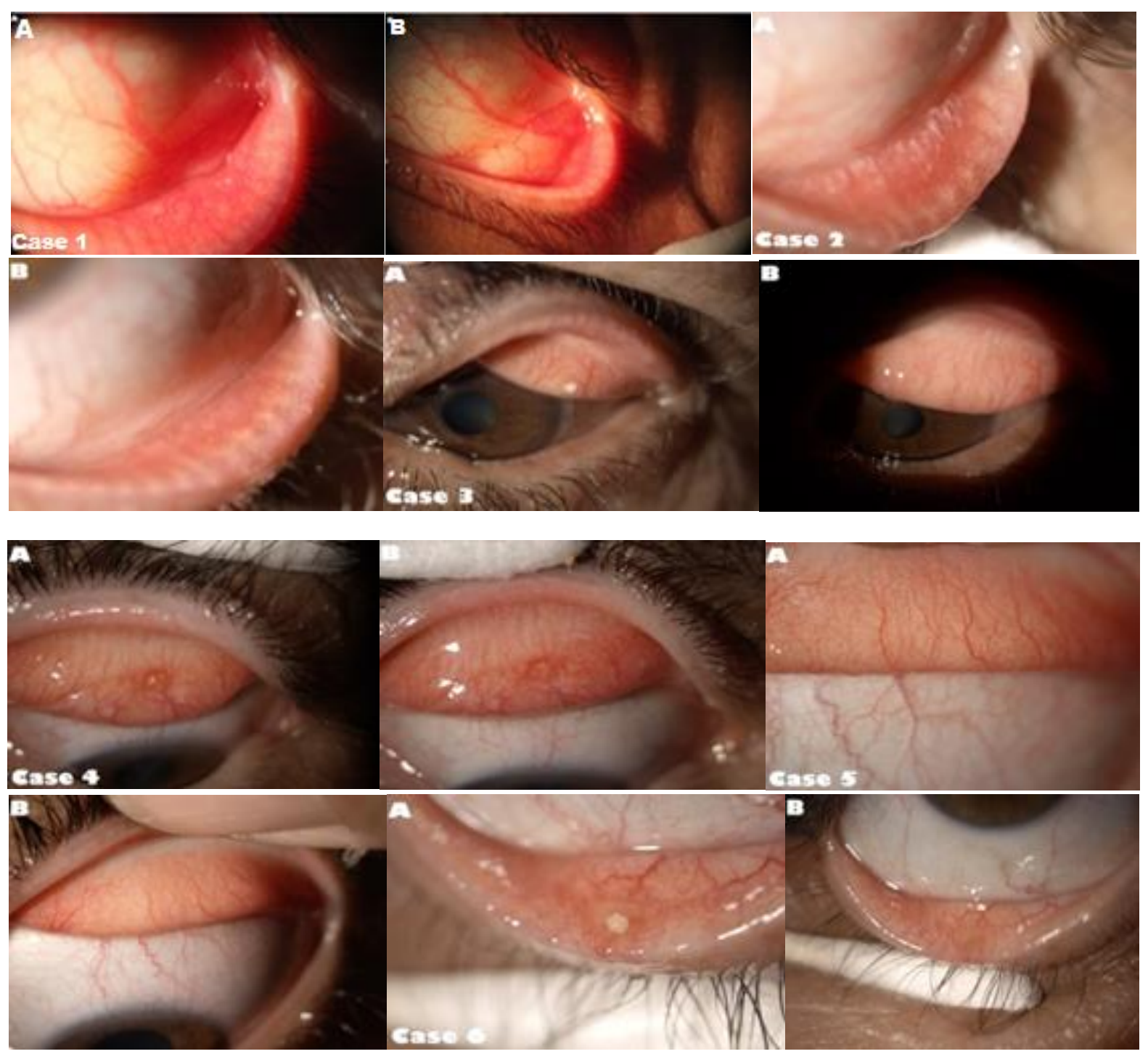

Figure 1: Treatment of conjunctival concretions using PANIS method. A: before treatment $\&$ B: after treatment

\section{Discussion}

The concretion is a relatively high prevalence disease that is more common in the elderly and has no gender superiority ${ }^{[3]}$. Although the disease is asymptomatic in most people, it can cause annoying symptoms if concretions erode through the epithelium ${ }^{[14]}$. People who complain of these symptoms are usually treated. The usual treatment method is using a $25 \mathrm{G}$ sterile needle and local anesthesia to extract lesions ${ }^{[4]}$. Although this method is inexpensive, it is not very pleasant and almost aggressive ${ }^{[4]}$. Plasma is one of the most recently used therapies in the treatment of diseases. This method is inexpensive, safe, does not require an operating room, and has a short learning curve. Sotiris et al. used this method for NonSurgical Blepharoplasty on 80 patients aged 40 to 78 years. Unlike classical Blepharoplasty, this method was without sutures, cuts, ectropion, entropion, slanted eyes, lagophthalmos, and other complications ${ }^{[15]}$. Rossi et al. also used this technique for Blepharoplasty in a pilot study. This study showed that after 30 to
45 days of follow-up, promising regenerative effects are seen in the collagen of the upper eyelid. The appearance of the patients has improved well, and no severe complications have been seen in the patients ${ }^{[16]}$. Nejat et al. have used this method to treat other eye diseases such as Conjunctivochalasis, Dry eye disease, Pinguecula and Conjunctival Cyst ${ }^{[10-13]}$. This method has already been tested in animal experiments by this team and it was confirmed in two studies on rabbits with a one-month and six-month follow-up that this method is safe ${ }^{[8,9]}$. Using this method in treating conjunctivochalasis in a six-month follow-up has shown that all six patients have shifted to a lower grade. No specific complications or complaints have been reported from patients ${ }^{[10]}$. The results of Conjunctival Cyst treatment with this method have shown that no complications were seen in patients during and after treatment. With one-day, one-week, and one- to six-month follow-up, this study has demonstrated that patients' cysts have completely healed and no recurrence observed ${ }^{[11]}$. Using this method in punctal occlusion seems to be a temporary practical approach for treating 
dry eye disease ${ }^{[13]}$. In pinguecula treatment, the study showed that (PANIS method) is effective, and after six months of follow-up, no recurrence and complications were observed ${ }^{[12]}$. Based on these studies, the PANIS method seems suitable and effective for treating conjunctival concretions. This procedure is less invasive than needle surgery. In the needle method, however, it is cheap but damages the surrounding tissues. In the PANIS method, the treatment is targeted, and minimal damage is done to the surrounding tissue. This method is fast and straightforward, has a good recovery with a low recurrence rate, and is Office based. Due to the multi-purpose use of this device in other treatments, it seems to have economic and practical justification in the long run $[10,11,15,16]$. Nevertheless, the present study is a case series, and to compare this method with other treatments, a clinical trial is needed. Follow-up in this study is six months. Longer follow-up and more patients can give us more information about the effectiveness of this treatment.

\section{Conclusions}

PANIS technique is an economical, easy, fast, safe method with shorter recovery time can effectively treat the Conjunctival concretions. However, further studies are needed with more patients and a long-term follow-up.

\section{Ethics approval and consent to participate}

We considered the tenets of the Declaration of Helsinki at all stages of the study. The protocol of the study was approved by the Semnan University of Medical Sciences. The study was registered in the Iranian Registry of Clinical Trials (IR.SEMUMS.REC.1398.318) and conducted at the Vision Health Research Center, Tehran, Iran, from 2019 - 2021. After receiving a complete description of the treatment process, the available alternatives, and the related risks, we obtained informed consent from all patients.

\section{List of abbreviations}

PANIS: Plasma assisted noninvasive surgery

BCVA: Best Corrected Visual Acuity

UCVA: Uncorrected Visual Acuity

OSDI: Ocular Surface Disease Index

IOP: Intraocular pressure

\section{Data Availability}

The datasets generated during and/or analyzed during the current study are available from the corresponding author on reasonable request. All data generated or analyzed during this study are included in this published article (and its supplementary information files)

\section{Conflicts of Interest}

The authors certify that they have NO affiliations with or involvement in any organization or entity with any financial interest (such as honoraria; educational grants; participation in speakers' bureaus; membership, employment, consultancies, stock ownership, or other equity interest; and expert testimony or patentlicensing arrangements), or non-financial interest (such as personal or professional relationships, affiliations, knowledge or beliefs) in the subject matter or materials discussed in this manuscript.

\section{Funding Statement}

This research did not receive any specific grant from funding agencies in the public, commercial, or not-for-profit sectors.

\section{Authors' contributions}

F N: design of the work, visiting diseases and treatment $\mathrm{Kh}$ J: contributing in Data collection

Sh E: have drafted the work or substantively revised it, contributing in Data collection

N-S N: contributing in Data collection

P M: Conceived and designed the analysis, contributing in Study design

All authors approved the submitted version (and any substantially modified version that involves the author's contribution to the study); All authors to have agreed both to be personally accountable for the author's own contributions and to ensure that questions related to the accuracy or integrity of any part of the work, even ones in which the author was not personally involved, are appropriately investigated, resolved, and the resolution documented in the literature.

\section{Acknowledgments}

Not applicable

\section{Supplementary Materials}

Sublimating concretions with plasma (white hand piece of the Plexr device).

\section{References}

[1] Kulshrestha M, Thaller V. Prevalence of conjunctival concretions. Eye. 1995;9(6):797-8.

[2] Sood G, Patel B. Conjunctival concretions. StatPearls. 2021.

[3] Haicl P, Janková H. Prevalance of conjunctival concretions. Ceska a slovenska oftalmologie: casopis Ceske oftalmologicke spolecnosti a Slovenske oftalmologicke spolecnosti. 2005;61(4):260-4.

[4] Lam D, Wong EL, Agar A, Coroneo MT, Francis IC. Curettage for Copious Conjunctival Concretions. Cureus. 2020;12(11).

[5] Amiel H, Koch PS. Tetracaine hydrochloride $0.5 \%$ versus lidocaine $2 \%$ jelly as a topical anesthetic agent in cataract surgery: comparative clinical trial. Journal of Cataract \& Refractive Surgery. 2007;33(1):98-100.

[6] Schlegel J, Köritzer J, Boxhammer V. Plasma in cancer treatment. Clinical Plasma Medicine. 2013;1(2):2-7.

[7] Metelmann H-R, Seebauer C, Miller V, Fridman A, Bauer G, Graves DB, et al. Clinical experience with cold plasma in the treatment of locally advanced head and neck cancer. Clinical Plasma Medicine. 2018;9:6-13.

[8] Nejat F, Nabavi N-S, Nejat M-A, Aghamollaei H, Jadidi K. Safety evaluation of the plasma on ocular surface tissue: an animal study and histopathological findings. Clinical Plasma Medicine. 2019;14:100084.

[9] Nejat F, Jadidi K, Amoli FA, Bagheri S, Aghamollaei H, Nejat M-A, et al. Safety evaluation of the atmospheric low-temperature plasma (ALTP) on the conjunctiva: an 
animal study and histopathological findings; 6-month follow-up. BMC ophthalmology. 2021;21(1):1-7.

[10] Jadidi K, Nabavi N-S, Nejat MA, Aghamollaei H, Adnani S-Y, Nejat B, et al. Evaluation of plasma assisted noninvasive surgery (PANIS) as a new approach for the treatment of conjunctivochalasis; a clinical case series. Expert Review of Ophthalmology. 2021:1-6.

[11] Nejat F, Jadidi K, Pirhadi S, Adnani S-Y, Nabavi N-S, Nejat MA. A Novel Approach to Treatment of Conjunctival Cyst Ablation Using Atmospheric LowTemperature Plasma. Clinical Ophthalmology (Auckland, NZ). 2020;14:2525.

[12] Nejat F, Jadidi K, Nejat MA, Nabavi N-S, Adnani S-Y, Eghtedari S. A Novel Approach to Treatment of Pinguecula Using Atmospheric Low-temperature Plasma: A Clinical Case Series. American Journal of Clinical and Experimental Medicine. 2021;9(5):148-52.

[13] Nejat F, Jadidi K, Eghtedari S, Nabavi N-S. Evaluation of efficacy of plasma assisted noninvasive surgery (PANIS) as a novel approach for temporary punctal occlusion: a clinical case series. SCIREA Journal of Clinical Medicine. 2021;6(6):421-32.

[14] Generics BC, List JAP, Stores JA. Conjunctival Concretions: Introduction, Symptoms, Causes, Diagnosis, Management and Prevention-www. genericdrugscan. com.
[15] Sotiris TG, Nikolaos G, Irini G. New treatment with plasma exeresis for non-surgical blepharoplasty. EC Ophthalmology. 2017;5(4):156-9.

[16] Rossi E, Farnetani F, Trakatelli M, Ciardo S, Pellacani G. Clinical and confocal microscopy study of plasma exeresis for nonsurgical blepharoplasty of the upper eyelid: a pilot study. Dermatologic surgery. 2018;44(2):283-90.

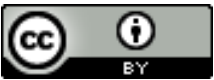

Open Access This article is licensed under a Creative Commons Attribution 4.0 International License, which permits use, sharing, adaptation, distribution and reproduction in any medium or format, as long as you give appropriate credit to the original author(s) and the source, provide a link to the Creative Commons license, and indicate if changes were made. The images or other third party material in this article are included in the article's Creative Commons license, unless indicated otherwise in a credit line to the material. If material is not included in the article's Creative Commons license and your intended use is not permitted by statutory regulation or exceeds the permitted use, you will need to obtain permission directly from the copyright holder. To view a copy of this license, visit https://creativecommons.org/licenses/by/4.0/.

(C) The Author(s) 2021 